\title{
ATIVIDADE ANTIOXIDANTE E FENOIS TOTAIS DE FRUTAS DE CAMPOS DOS GOYTACAZES RJ
}

\author{
Natalia Ribeiro Bernardes \\ Doutoranda em Produção Vegetal/Laboratório de Tecnologia de Alimentos/UENF/RJ \\ nataliar_bernardes@yahoo.com.br
}

\section{Simone Vilela Talma}

Mestranda em Produção Vegetal/Laboratório de Tecnologia de Alimentos/UENF/RJ

simonevtalma@yahoo.com.br

\section{Shalline Hermes Sampaio}

Mestranda em Produção Vegetal/Laboratório de Tecnologia de Alimentos/UENF/RJ

shafarmaceutica@hotmail.com.br

\section{Clara Reis Nunes}

Doutoranda em Produção Vegetal/Laboratório de Tecnologia de Alimentos/UENF/RJ clara_biol@yahoo.com.br

\section{Juliana Araujo Rangel de Almeida}

Mestranda em Produção Vegetal/Laboratório de Tecnologia de Alimentos/UENF/RJ

julianaara@yahoo.com.br

\author{
Daniela Barros de Oliveira \\ Doutora em Química de Produtos Naturais/UFRJ/RJ \\ Laboratório de Tecnologia de Alimentos/UENF/RJ \\ dbarrosoliveira@uenf.br
}

\section{RESUMO}

O consumo de frutas, hortaliças e condimentos têm aumentado principalmente em decorrência do seu valor nutritivo e dos seus efeitos terapêuticos. Esses alimentos apresentam substâncias que estão relacionadas com efeitos benéficos ao organismo humano, como a ação antioxidante, o que certamente vem contribuindo para uma contínua melhoria da saúde humana, com o retardo do envelhecimento e a prevenção de certas doenças. Entre as várias substâncias que têm sido consideradas responsáveis por essa proteção, podem ser citados alguns pigmentos como os carotenóides, betalaínas e os flavonóides (substâncias fenólicas). O objetivo deste trabalho foi avaliar a atividade antioxidante e o teor de fenóis totais na casca e polpa dos frutos: ameixa, laranja, maçã e kiwi que foram adquiridas em mercados de Campos dos Goytacazes-RJ. A atividade antioxidante foi avaliada pelo método do DPPH e a quantificação de compostos fenólicos foi realizada por Folin-Denis. Observou-se que a casca de todas as frutas avaliadas neste trabalho apresentaram conteúdos de fenóis totais superiores ao da polpa, sendo que o kiwi apresentou maiores valores $(1273,4 \mathrm{mg} / 100 \mathrm{~g}$ e 981,8 $\mathrm{mg} / 100 \mathrm{~g}$ para casca e polpa, respectivamente). Em relação a atividade antioxidante, a polpa e a casca de todas as frutas apresentaram valores acima de $85 \%$ de sequestro de radicais livres, exceto a laranja que apresentou $51,88 \%$ e $59,87 \%$ de sequestro de radicais livres para a casca e polpa, respectivamente. Contudo, os resultados obtidos neste trabalho fazem destas frutas uma fonte promissora de compostos antioxidantes, cujo cultivo e consumo deveriam ser estimulados no comércio local.

Palavras-chave: Fenóis, radicais livres, frutas in natura. 


\begin{abstract}
The consumption of fruits, vegetables and has increased mainly due to its nutritional and therapeutic effects. These foods have substances that are associated with beneficial antioxidant effects for human beings, contributing to the improvement of human health, delaying aging and preventing certain diseases. Among the various substances which responsible for such protection, some pigments such as carotenoids, flavonoids and betalaines (phenolic substances) may be cited. The aim of this study was to evaluate the antioxidant activity and total phenolic content of peel and pulp of plum, orange, apple and kiwi acquired in local markets of Campos dos Goytacazes-RJ. The antioxidant activity was evaluated by DPPH and quantification of phenolic compounds was performed by Folin-Denis method. It was observed that the peel all fruits studied in this work showed higher total phenol content of the pulp and kiwi showed that the highest values (981.8 $\mathrm{mg} / 100 \mathrm{~g}$ and $1273.4 \mathrm{mg} / 100 \mathrm{~g}$ for peel and pulp, respectively). Regarding the antioxidant activity, the pulp and peel all the fruit had values above $85 \%$ of scavenging free radicals, except orange which showed $51.88 \%$ and $59.87 \%$ of scavenging free radicals to the peel and pulp respectively. However, the present results of these fruits are a promising source of antioxidant compounds, whose cultivation and consumption should be encouraged in local trade.
\end{abstract}

Keywords: Natural products, free radicals, fresh fruit.

\title{
1. INTRODUÇÃO
}

O grande interesse no estudo dos antioxidantes é decorrente, principalmente, do efeito dos radicais livres no organismo. A oxidação é inerente à vida aeróbica e, dessa forma, os radicais livres são produzidos naturalmente. Essas moléculas geradas in vivo estão envolvidas na produção de energia, fagocitose, regulação do crescimento celular, sinalização intercelular e síntese de substâncias biológicas importantes (BARREIROS, DAVID e DAVID, 2006).

Além disso, os radicais livres reagem com DNA, RNA, proteínas e outras substâncias oxidáveis, promovendo danos que podem contribuir para o envelhecimento e a instalação de doenças degenerativas, como câncer, aterosclerose, artrite reumática, entre outras (LACHMAN et al.; 2010, MELO et al., 2006).

$\mathrm{O}$ excesso de radicais livres no organismo é combatido por antioxidantes produzidos pelo corpo ou absorvidos da dieta. Quando há um desbalanço entre a produção de radicais livres e os mecanismos de defesa antioxidante, ocorre o chamado "estresse oxidativo". Os radicais livres em excesso podem ser originados por defeitos na respiração mitocondrial, metabolismo do ácido araquidônico, ativação-inibição de sistemas enzimáticos ou por fatores exógenos, como poluição, hábito de fumar ou ingerir álcool, ou ainda, por uma nutrição inadequada (NÚÑEZ-SELLÉS, 2005).

A principal forma de obtenção pelo organismo de antioxidantes consiste na ingestão de compostos com esta atividade através da dieta. Os principais antioxidantes dietéticos são algumas vitaminas, compostos fenólicos e carotenoides. Os antioxidantes agem interagindo com os radicais livres antes que estes possam reagir com as moléculas biológicas, evitando que ocorram as reações em cadeia ou prevenindo a ativação do oxigênio a produtos altamente reativos (BERNARDES et al., 2010; RATNAM et al., 2006).

Os antioxidantes podem ser divididos em duas classes: os com atividade enzimática e os não enzimáicos. No primeiro grupo estão os compostos capazes de inibir a iniciação da oxidação, ou seja, as enzimas que removem as espécies reativas ao oxigênio. No segundo grupo estão às moléculas que interagem com as espécies radicalares e são consumidas durante a reação. Neste grupo incluem-se os compostos fenólicos (MARIOD et al.; 2009; MOREIRA e MANCINI FILHO, 2003).

Os compostos fenólicos são substâncias amplamente distribuídas no reino vegetal, em particular nos frutos e em outras partes dos vegetais. São moléculas heterogêneas que apresentam em sua estrutura vários grupos benzênicos característicos, substituídos por grupamentos hidroxilas (OLIVEIRA et al., 2009; ANGELO et al., 2007; HERNÁNDEZ \& PRIETO GONZÁLES,1999). 
Essas substâncias agem como antioxidantes não somente pela sua habilidade em doar hidrogênio ou elétrons, mas também por causa de seus radicais intermediários estáveis, que impedem a oxidação de vários ingredientes do alimento, particularmente de ácidos graxos e de óleos (ALI et al., 2009; CUVELIER et al.,1992 ; MAILLARD et al.,1996).

Desta forma, a importância da pesquisa por antioxidantes naturais tem aumentado muito nos últimos anos. Compostos típicos que possuem atividade antioxidante incluem a classe de fenóis, ácidos fenólicos e seus derivados, flavonóides, tocoferóis, fosfolipídios, aminoácidos, ácido fítico, ácido ascórbico, pigmentos e esteróis (OLIVEIRA et al., 2009; ANGELO et al., 2007; JAYAPRAKASHA, 2000). O objetivo deste trabalho foi avaliar a atividade antioxidante e determinar os compostos fenólicos totais das cascas e polpas de diferentes frutas amplamente comercializadas em Campos dos Goytacazes - RJ, sendo pioneiro por informar os consumidores do município acerca das propriedades benéficas destas frutas uma vez que estas fazem parte da economia local.

\section{MATERIAL E MÉTODOS}

\subsection{Amostras}

Frutos de Malus domestica Borkh (maçã), Citrus sinensis (laranja), Prunus sp. (ameixa) e Actinidia deliciosa (kiwi) foram adquiridos maduros em supermercados do comércio local de Campos dos Goytacazes (RJ), no mês de março de 2010 e em seguida foram levados ao Setor de Química de Alimentos do Laboratório de Tecnologia de Alimentos (LTA), do Centro de Ciências e Tecnologias Agropecuárias (CCTA) da Universidade Estadual do Norte Fluminense Darcy Ribeiro (UENF) para a avaliação da atividade antioxidante e determinação de fenóis totais. Todas as frutas foram avaliadas no mesmo dia em que foram adquiridas, sendo realizadas três repetições, onde cada fruta correspondeu a uma repetição e cada análise realizada em triplicata.

\subsection{Metodologia de Extração das Amostras}

A polpa e a casca dos frutos $(500 \mathrm{mg}$ ) foram macerados em gral e pistilo com uma solução de acetona/ água (7:3) para a obtenção do extrato. Em seguida, este foi utilizado para as análises da avaliação da atividade antioxidante e determinação do teor de fenóis totais.

\subsection{Avaliação da Atividade Antioxidante}

A avaliação da atividade antioxidante foi realizada pelo método fotocolorimétrico do radical livre estável DPPH (1,1-difenil-2-picrilidrazila). Este método se baseia no sequestro do radical 1,1-difenil-2picrilhidrazila (DPPH) pelos antioxidantes, que produz uma diminuição de absorção em $515 \mathrm{~nm}$. Quando uma solução de DPPH é misturada com uma substância que pode doar um átomo de hidrogênio, a forma reduzida do radical gerado é acompanhada de perda de cor (ALI et al.; 2009).

Essa técnica consiste em adicionar $1 \mathrm{~mL}$ do extrato em concentrações que variam de $0,1-1000$ $\mu \mathrm{g} / \mathrm{mL}$. A este foi adicionado $1 \mathrm{~mL}$ de uma solução metanólica de DPPH $(0,1 \mathrm{mM})$, a reação foi processada em 1 hora à temperatura ambiente. Imediatamente, a absorção do DPPH foi verificada em $515 \mathrm{~nm}$ em um espectrofotômetro UV-VIS Shimadzu Mini 1240. Todos os experimentos foram realizados em triplicata.

A capacidade de sequestrar radical livre foi expressa como percentual de inibição de oxidação do radical e calculado conforme fórmula abaixo:

\section{$\%$ Inibição $=\left(\left(\mathrm{A}_{\mathrm{DPPH}}-\mathrm{A}_{\mathrm{Extr}}\right) / \mathrm{A}_{\mathrm{DPPH}}\right) * 100$}

Onde ADPPH é a absorbância da solução de DPPH e $A_{\text {Extr }}$ é a absorbância da amostra em solução (ROESLER, et al., 2007).

\subsection{Determinação de Fenóis Totais}

A quantificação dos fenóis totais foi realizada através do método de Folin-Denis, o qual envolve a redução do reagente por compostos fenólicos da amostra com a formação de um complexo azul no qual a sua intensidade aumenta linearmente a $760 \mathrm{~nm}$ (SWAIN e HILLLS, 1959). Em 0,5 mL de extrato foram adicionados $0,5 \mathrm{~mL}$ do reagente de Folin-Denis. Posteriormente, adicionou-se $3 \mathrm{~mL}$ de água destilada e após 
1 hora, $1 \mathrm{~mL}$ da solução de carbonato de cálcio saturada foi adicionada. A leitura foi realizada em espectrofotômetro a $760 \mathrm{~nm}$. Foi construída uma curva padrão com o ácido tânico em diferentes concentrações ( 2 a $100 \mu \mathrm{g} / \mathrm{mL}$ ) e a partir destes resultados foi possível calcular a concentração das amostras analisadas.

\subsection{Análises estatísticas}

Os dados foram submetidos à análise da variância (ANOVA, $\mathrm{p}<0,05)$ e teste de média Tukey $(\mathrm{p}<$ $0,05)$.

As análises estatísticas foram realizadas utilizando-se o programa estatístico SAS (2003) - Statistical Analysis System, versão 9,3.

\section{RESULTADOS E DISCUSSÃO}

Na Tabela 1 encontram-se os valores obtidos na avaliação da atividade antioxidante, bem como a quantificação estimada da concentração de fenóis totais das polpas e cascas das frutas avaliadas a partir da curva padrão.

Tabela 1 - Atividade antioxidante e conteúdo de fenóis totais das polpas e cascas das frutas avaliadas.

\begin{tabular}{ccc}
\hline Frutas Avaliadas & $\begin{array}{c}\text { Atividade Antioxidante }^{\mathbf{1}}(\mathbf{\%} \text { de sequestro } \\
\text { de radicais livres) }^{\text {and }}\end{array}$ & $\begin{array}{c}\text { Fenóis Totais }^{1} \\
(\mathbf{m g} / \mathbf{1 0 0 g})\end{array}$ \\
\hline Ameixa casca & $92.84^{\mathrm{a}}$ & $825,95^{\mathrm{c}}$ \\
Ameixa polpa & $92.56^{\mathrm{a}}$ & $247,09^{\mathrm{e}}$ \\
Maçã casca & $92.74^{\mathrm{a}}$ & $328,55^{\mathrm{e}}$ \\
Maçã polpa & $88.30^{\mathrm{a}}$ & $212,94^{\mathrm{e}}$ \\
Laranja casca & $51.88^{\mathrm{b}}$ & $472,78^{\mathrm{d}}$ \\
Laranja polpa & $59.87^{\mathrm{b}}$ & $316,36^{\mathrm{e}}$ \\
Kiwi casca & $95.69^{\mathrm{a}}$ & $1273,41^{\mathrm{a}}$ \\
Kiwi polpa & $96.09^{\mathrm{a}}$ & $981,87^{\mathrm{b}}$ \\
\hline
\end{tabular}

${ }^{1}$ Médias com letras iguais na mesma coluna não diferem significativamente a $p<0,05$, segundo o teste de Tukey.

De acordo com a Tabela 1, observa-se que as cascas de todos os frutos apresentaram conteúdos de fenóis totais significativamente $(\mathrm{p}<0,05)$ superiores ao da polpa, exceto para a maçã que não apresentou diferença significativa entre o conteúdo fenólico presente na casca e na polpa e que não diferiu também da polpa da ameixa e da laranja. Dentre os frutos avaliados, o kiwi apresentou o maior conteúdo fenólico tanto para polpa quanto para a casca, diferindo significativamente $(\mathrm{p}<0,05)$ das demais frutas analisadas. Embora, a casca desta fruta não seja comestível in natura, tal resultado sugere outras formas de utilização para o seu consumo, bem como para a casca da laranja.

Em relação à atividade antioxidante, verifica-se que as frutas avaliadas apresentaram valores superiores a $80 \%$ de sequestro de radicais livres, exceto para a laranja que apresentou $51,88 \%$ e $59,87 \%$ para a casca e polpa, respectivamente (Tabela 1$)$, diferindo significativamente $(p<0,05)$ das outras. No kiwi, apesar de não apresentar diferença significativa entre a ameixa e a maçã, tanto para a casca quanto para a polpa, observa-se que sua atividade de sequestro de radicais livres apresentaram resultados expressivos, bem como uma correlação positiva com o conteúdo de fenóis totais.

Uma vez que estas frutas apresentam grande importância na população local, por serem amplamente consumidas, estas são produzidas em outros estados e trazidas sob temperatura ambiente para a comercialização local, embora permaneçam armazenadas sob refrigeração até a sua exposição para a venda, exceto a laranja. Dessa forma, sugerem-se estudos mais específicos para verificar se esses conteúdos se mantêm ao longo da vida de prateleira, a qual pode vir a afetar os resultados obtidos.

A cor característica da ameixa decorre da presença de antocianinas, o que justifica uma concentração considerável de fenóis em relação aos outros frutos (LIMA et al., 2000).

LIU et al.(2002) encontraram valores de 359,2 a $512,7 \mathrm{mg} / 100 \mathrm{~g}$ de fenóis totais em diversas variedades de framboesa enquanto, KIM et al.(2003) encontraram valores de 174 a $375 \mathrm{mg} / 100 \mathrm{~g}$ em 
diversas variedades de acerola e relatou uma valor próximo a $118 \mathrm{mg} / 100 \mathrm{~g}$ em maçã 'Gala'. Observa-se que o resultado encontrado de fenóis totais na polpa da maçã neste trabalho $(212,94 \mathrm{mg} / 100 \mathrm{~g})$ foi superior que o encontrado por KIM et al. (2003). Tal fato pode ser explicado devido à variabilidade entre os tipos de cultivares. Embora as maçãs apresentem baixas taxas de fenólicos totais quando comparada as outras frutas, observa-se que os polifenóis isolados da polpa de maçã apresentam forte atividade antioxidante no sistema $\beta$ caroteno ácido linoleico e no sequestro do DPPH (PEARSON et al., 1999). Estes autores demonstraram que os fenólicos presentes em suco comercial e extrato fresco de maçãs (casca, polpa e fruta inteira) inibiram, in vitro, a oxidação de LDL humana.

Ao contrário dos resultados apresentados na Tabela 1, a literatura relata que as diferentes variedades de laranja têm além do reconhecido valor nutricional, teores relativamente elevados de substâncias com atividade antioxidante, tais como o ácido ascórbico e diversos compostos fenólicos, aos quais se atribui um importante papel na prevenção de doenças crônico-degenerativas (GARDENER et al., 2000).

Segundo HEIM et al. (2002) os compostos fenólicos são os maiores responsáveis pela atividade antioxidante em frutos. Embora, a vitamina $\mathrm{C}$ seja considerada como o maior contribuinte na atividade antioxidante, SUN et al. (2002) demonstraram que sua contribuição para esta atividade é baixa e afirmaram que a maior contribuição para o sequestro de radicais livres em frutos se deve à presença de compostos fitoquímicos, como os flavonoides e os carotenoides, em sua composição.

Nesse contexto, sabe-se que os hábitos alimentares da população brasileira são bastante diversificados. No entanto, de modo geral, a ingestão diária de flavonóide (cerca de $23 \mathrm{mg} / \mathrm{dia}$ ) excede àquela de outros antioxidantes como a de $\beta$-caroteno $(2-3 \mathrm{mg} / \mathrm{dia})$ e vitamina $E(7-10 \mathrm{mg} / \mathrm{dia})$ conforme relatado por HERRMANN (2002). Assim sendo, os flavonóides representam uma contribuição importante ao potencial antioxidante da dieta. Estudos epidemiológicos mostraram haver uma relação entre o consumo de frutas e verduras e a proteção contra vários tipos de câncer (BLOCK, 1992; ZEIGER, 1991) doenças isquêmicas (FALLER e FIALHO, 2009) e diabetes (COLDITZ, 1992). Embora outros estudos sejam necessários, principalmente acerca do mecanismo de ação, absorção, metabolização, excreção e toxicidade, pode-se dizer que as frutas analisadas neste trabalho podem trazer efeitos benéficos ao organismo humano, o que já foi demonstrado em diversos trabalhos (FIGUEIREDO et al., 2008; ANJO, 2004; GRAMEZZI et al, 1990).

\section{CONCLUSÃO}

Todas as frutas avaliadas apresentaram a presença de compostos fenólicos e atividade antioxidante, tanto na casca quanto na polpa, com destaque para o kiwi, fazendo deste trabalho de extrema importância tanto para a economia local quanto para a informação aos consumidores. Além disso, as cascas dos frutos apresentaram uma concentração maior de fenóis totais quando comparados com a polpa o que sugere outras formas de utilização para o seu consumo.

Pode-se dizer que as frutas analisadas podem trazer efeitos benéficos ao organismo humano, mas faz-se necessário estudos mais aprofundados para verificar a contribuição de cada fruta para o organismo bem como a quantidade necessária, além de verificar se esses conteúdos se mantém ao longo da vida de prateleira e em diferentes formas de armazenamento.

\section{REFERÊNCIAS BIBLIOGRÁFICAS}

ALI, S. S.; KASOJU, N.; LUTHRA, A.; SINGH, A.; SHARANABASAVA, H.; SAHU, A.; BORA, U.Indian medicinal herbs as sources of antioxidants. Food Research International 41:1-15, 2009.

ANGELO, P. M.; JORGE, N. Compostos fenólicos em alimentos - Uma breve revisão. Rev. Inst. Adolfo Lutz, v. 66, n. 1, p. 1-9, 2007.

ANJO, D. L. C. Alimentos funcionais em angiologia e cirurgia vascular. Jornal Vascular Brasileiro, v. 3, n. 2, p. 145-154, 2004.

BARREIROS, A. L.; DAVID, J. M.; DAVID, J. P. Estresse oxidativo: relação entre geração de espécies reativas e defesa do organismo. Química Nova, v. 29, n. 1, p. 113-123, 2006. 
BERNARDES, N. R.; PESSANHA, F. F.; OLIVEIRA, D. B. Alimentos Funcionais: Uma breve revisão. Ciência e Cultura - Revista Científica Multidisciplinar do Centro Universitário da FEB, v.6, n. 2. Novembro, 2010.

BLOCK, G.; PATTERSON, B.; SUBAR, A. Fruit, vegetables and cancer prevention: a review of the epidemiological evidence. Nutr. Cancer, v. 17, p. 1-29, 1992.

COLDITZ, G.A.; MANSON, J. E.; STAMFER, M. J.; ROSNER, B.; WILLETT, W.; SPIEZER, F. E. Diet and risk of clinical diabetes in women. Am. J. Clin. Nutr., v. 55, p. 118-23, 1992.

CUVELIER, M. E.; RICHARD, H.; BERSET, C. Comparison of the antioxidant activity of some acid phenols: structure-activity relationship. Bioscience Biotechnology and Biochemistry, Benkyoku, v.59, p.324325,1992 .

FALLER, A. L. K.; FIALHO, E. Frutas, hortaliças e disponibilidade de polifenóis no Brasil. Revista de Saúde Pública, v. 43, p. 211-218, 2009.

FIGUEIREDO, I. C. R.; JAIME, P. C.; MONTEIRO, C. A. Fatores associados ao consume de frutas, legumes e verduras em adultos da cidade de São Paulo. Rev Saúde Pública, v. 42, n. 5, p. 777-85, 2008.

GARDENER, P. T.; WHITE, T. A. C.; McPHAIL, D. B.; DUTHIE, G. G. The relative contributions of vitamin $\mathrm{C}$, carotenoids and phenolics to the antioxidant potencial of fruit juices. Food Chem., v.68, p.471474, 2000 .

GRAMEZZI, A.; GENTOLA, A.; FASOLI, M. Assossiation between certain food and risk of acute myocardial infartion in women. Br. Med. J.,v. 300, p. 771-773, 1990.

HEIM, K.E.; TAGLIAFERRO, A.R.; BOBILYA, D.J.. Flavonoid antioxidants: chemistry, metabolism and structure-activity relationships. J Nutr Biochem, v.13, p.572-584, 2002.

HERNÁNDEZ, A.M.; PRIETO GONZÁLES, E.A. Plantas que contienen polifenoles. Revista Cubana de Investigaciones Biomedica, Ciudad de la Habana v.18, n.1, p. 12-14,1999.

HERRMANN, M.S. Aspectos nutricionais dos flavonóides. In: Estresse oxidativo e antioxidantes. Porto Alegre: Ed. Ulbra, 2002. p.105-119.

JAYAPRAKASHA, F.K.; JAGANMOHAN RAO, L. Phenolic constituents from lichen Parmotrema stuppeum (Nyl.). Hale and their antioxidant activity. Z. Naturforsch, v. 55C, p. 1018-1022, 2000.

KIM, D.; JEONG, S.W.; LEE, C.Y. Antioxidant capacity of phenolic phytochemicals from various cultivars of plums. Food Chemistry, Kidlington, v.81, p.231-326, 2003.

LACHMAN, J., ORSAK, M., HEJTMANKOVA, A., KOVAROVA, E. Evaluation of antioxidant activity and total phenolics of selected Czech honeys. Food Science and Technology, v. 43, p. 52-58, 2010.

LIMA, V.L.A.G de; MÉLO, E. de A.; LIMA, L. dos S.; NASCIMENTO, P.P. do. Caracterização físicoquímica e sensorial de pitanga roxa. Revista Brasileira de Fruticultura, v.22, p.382-385, 2000.

LIU, M.; LI, X.Q.; WEBER, C.; LEE YONG, C.; BROWN, J.; LIU, R.H. Antioxidant and anti proliferative activities of raspberries. Journal of Agricultural and Food Chemistry, Washington, v.50, p. 2926-2930, 2002.

MAILLARD, M.N.; SOUM, M.H.; BOIVIA, P.; BERSET, C. Antioxidant activity of barley and malt: relationship with phenolic content. Lebensmittel-Wissenschaft \& Technologie, London, v. 3, p. 238244,1996 . 
MARIOD, B. A., IBRAHIM, R. M., ISMAIL, M., ISMAIL, N. Antioxidant activities of phenolic rich fractions (PRFs) obtained from black mahlab (Monechma ciliatum) and white mahlab (Prunus mahaleb) seedcakes. Food Chemistry, v. 118, p. 120-127, 2009.

MELO, E.; MACIEL, M.; LIMA, V.; LEAL, F.; CAETANO, A.; NASCIMENTO, R. Capacidade antioxidante de hortaliças usualmente consumidas. Ciência Tecnologia Alimentos, v. 26, n. 3, p. 639-644, 2006.

MOREIRA, A.V.B.; MANCINI- FILHO, J. Atividade antioxidante das especiarias mostarda, canela e ervadoce em sistemas aquoso e lipídico. Nutrire, São Paulo, V.25, p.31-46, 2003.

NÚÑEZ-SELLÉS, A.J. Antioxidant Therapy: Myth or Reality?. J. Braz. Chem. Soc., v. 16, n. 4, p. 699-610, 2005.

OLIVEIRA, D. B.; PESSANHA, N. N. C.; BERNARDES, N. R.; SILVA, W. D.; MUZITANO, M. F.; OLIVEIRA, D. R. Extrato dos frutos de Cereus Fernambucensis: Atividade Antioxidante e Inibição da Produção de Óxido Nítrico (NO) por Macrófagos. Interscienceplace, ano 2, n. 07, Maio/Junho, 2009.

PEARSON, D.A.; TAN, C.H.; GERMAN, J.B.; DAVIS, P.A.; GERSHWIN, M.E. Apple juice inhibits human low density lipoprotein oxidation. Life Sciences, v.64, p.1913-1920, 1999.

RATNAM, D.; ANKOLA, D.; BHARDWAJ, V.; SAHANA, D.; KUMAR, M. Role of antioxidants in prophylaxis and therapy: A pharmaceutical perspective. J. Control Release., v. 113, n. 2, p. 189-207, 2006.

ROESLER, R.; MALTA, L.G.; CARRASCO, L.C.; HOLANDA, R.B.; SOUSA, C.A.S.; PASTORE, G.M. Atividade Antioxidante de frutas do cerrado. Ciência Tecnologia Alimentos, Campinas. v. 27, n.1, p. 53-60, 2007.

SAS ${ }^{\circledR}$ Statistical Analysis System, SAS Institute Inc., SAS User’s Guide, Cary, USA: SAS Inst., 2003.

SUN, J. CHU, Y.; WU, X.; LIU, R.H. Antioxidant and antiproliferative activities of common fruits. J. Agric Food Chem, v.50, p.7449-7454, 2002.

SWAIN, T.; HILLIS, W.E. The phenolics constituents of prumus domestica: the quantitative analysis of phenolic constituents. Journal of Science Food Agriculture, v. 10, p. 63-8, 1959.

ZEIGER, R. Vegetables, fruits and carotenes and risk of cancer.Am. J. Clin. Nutr.,v.53, suppl. p. 251S-259S, 1991. 